\title{
Encapsulation of Viable Aerobic Microorganisms in Silica Gels
}

\author{
ANTHONY P. TAYLOR, ${ }^{*}$ KIM S. FINNIE, JOHN R. BARTLETT AND PETER J. HOLDEN \\ Australian Nuclear Science and Technology Organisation (ANSTO), Lucas Heights, Sydney, Australia \\ apt@ansto.gov.au
}

\begin{abstract}
The aim of this study was to develop techniques to sterilise the surface of silica gels containing encapsulated cells and the liquid broth they were immersed in, so that the observed metabolic activities could be unambiguously assigned to fully encapsulated cells. Gel surfaces were sterilised by UV-irradiation daily. The surfaces of the gels and the overlaying medium remained sterile for 20 days following irradiation, as demonstrated by the lack of visible surface growth and viable cells in the medium. We report the encapsulation of a viable, metabolically active, aerobic fungus Penicillium chrysogenum, and the aerobic Gram-positive bacterium Streptomyces rimosus in gels derived from aqueous silica sols. Carbohydrate consumption (catabolism) and antibiotic biosynthesis (penicillin or oxytetracycline) (anabolism) were monitored in both cultures, demonstrating that the encapsulated cells remained viable within the gel matrix. This demonstrates that the silica gels are sufficiently porous to sustain metabolic activities of aerobic cells, which require the diffusion of oxygen and other substrates within the gel's nanopore network.
\end{abstract}

Keywords: bioencapsulation, sol-gel, Penicillium chrysogenum, Streptomyces rimosus, penicillin, oxytetracycline, UV-sterilisation

\section{Introduction}

A variety of cell-immobilisation techniques using porous solid phases to anchor the biomass or to sediment cells from a nutrient broth have been investigated [1]. Reports of bioencapsulation of microorganisms in host matrices have included sol-gel derived materials [2-5]. However, there has been a notable absence of procedures to ensure that the biocatalysis data acquired was only from cells encapsulated within the gel, and little emphasis has been placed on preventing outgrowth of cells into the bulk liquid phase $[3,6,7]$. The aim of this study, was to develop techniques to sterilise the surface of gels and the liquid medium they were immersed in, so that metabolic activities could be unambiguously assigned to fully encapsulated cells only. The fungus Penicillium chrysogenum and the bacterium Streptomyces rimosus are used in industry to produce penicillin and oxytetracycline, respectively, and are the most studied of the antibiotic biosynthesis-

\footnotetext{
*To whom all correspondence should be addressed.
}

ing microbes. $S$. rimosus has also been widely studied in solid-state oxytetracycline biosynthesis $[8,9]$. This paper reports the efficacy of UV-irradiation as a method for sterilising both the surface of microbe-doped silica gels, and the liquid growth medium. Selective sterilisation enabled the comparison of carbohydrate consumption and antibiotic biosynthesis activity by cells encapsulated within the silica gel with activity due to cells immobilised on the external surface of the gel and those freely suspended in liquid phase. The choice of aerobic microorganisms for these trials also provided an opportunity to establish whether sufficient oxygen is available to cells encapsulated within gels to promote growth and normal biological function.

\section{Experimental}

\subsection{Cultures and Media}

Penicillium chrysogenum (DSMZ 895) and Streptomyces rimosus subspecies rimosus (DSMZ 40260) 
were used as test organisms in the biosynthesis assays and Bacillus subtilis (ATCC 6633) was used to test antibiotic production in the disc-diffusion assays.

P. chrysogenum nutrient broth (PCB) contained (g/l): malt extract, 3.0; yeast extract, 1.5; glucose, 10.0; and peptone, 1.5. Wickerham's malt yeast extract broth and agar (MYEB and MYEA) contained (g/l): malt extract, 3.0; peptone, 5.0; yeast extract, 3.0; glucose, 10.0; and in MYEA agar, 20.0. Peptone yeast extract agar (PYEA) contained (g/l): peptone, 10.0; yeast extract, 5.0; $\mathrm{NaCl}, 5.0$; and agar, 20.0. The $\mathrm{pH}$ of the PYEA was adjusted to 7.2. Agar plates were poured each with $25 \mathrm{ml}$ of PYEA. S. rimosus broth (SRB) contained (g/l): soluble starch, 10.0; yeast extract, 1.0; beef extract, 1.0; tryptone, 2.0 ; and $\mathrm{FeSO}_{4} \cdot 7 \mathrm{H}_{2} \mathrm{O}, 0.1$. The supernatant was used for the assay, to exclude any starch solids which may inhibit surface sterilisation. All media were autoclaved at $121^{\circ} \mathrm{C}$ for $16 \mathrm{~min}$ prior to use.

\subsection{Petri Dish Bioreactors (PDB) and Silica Gels}

Petri dish bioreactors (PDB) were prepared by casting silica gels, inoculated with spores, in petri dishes followed by irrigation with appropriate liquid growth media as described below. Falcon Optilux ${ }^{\mathrm{TM}}$ plastic petri dishes $(100 \mathrm{~mm} \times 20 \mathrm{~mm})$ were etched for $6.0 \mathrm{~min}$ in water plasma at $\approx 5.0 \times 10^{-2}$ millibar in a $40 \mathrm{~W}$ RF-plasma generator operating at $13.56 \mathrm{mH} \mathrm{RM}$, to hydroxylate the surfaces and improve their wettability. Colloidal silica solution (Bindzil ${ }^{\mathrm{TM}}$, Eka Chemicals, $\mathrm{pH}$ 10) was sterilized via $\gamma$-irradiation, and subsequently adjusted to $\mathrm{pH} 6$ (using universal indicator and $4.0 \mathrm{M} \mathrm{HCl}$ ), to initiate gelation. In initial preparative trials, cells and spore suspensions were immediately added to the silica, but they were buoyant in the dense $(1.213 \mathrm{~g} / \mathrm{ml})$ solution, which resulted in concentration of the biomass on the gel surface only. Most of the biomass was subsequently killed on exposure to UV irradiation. Two techniques were used to overcome this problem:

1. in the penicillin biosynthesis assay, $P$. chrysogenum spores were grown on a thin layer of gel before the hyphae were encapsulated in a thicker overlying layer of gel; and

2. in the oxytetracycline biosynthesis assay, S. rimosus spores were encapsulated in a thin layer of gel, which was overlayed with a thicker layer of gel before culture.
In the penicillin biosynthesis assay, two PDB (PDB1 and PDB2) were assembled in duplicate with a $0.5 \mathrm{~mm}$ thick gel $(2.95 \mathrm{ml})$ containing $1.0 \times 10^{6} \mathrm{cfu} / \mathrm{ml}$ of $P$. chrysogenum spores $\left(3.0 \times 10^{6} \mathrm{cfu} / \mathrm{PDB}\right)$. The gels were aged overnight to prevent redispersion and were cultured for two days at $26^{\circ} \mathrm{C}\left( \pm 0.1^{\circ} \mathrm{C}\right)$ submerged in $11.8 \mathrm{ml}(2.0 \mathrm{~mm}$ thick $)$ of PCB. The hyphae that grew in the liquid medium were then encapsulated in a layer of silica gel $1.0 \mathrm{~mm}$ thick $(5.9 \mathrm{ml})$. The buoyant cells were tethered to the lower gel layer, so the hyphae stood up and were relatively evenly distributed throughout the upper layer of gel. The gel was aged overnight and then covered with $11.8 \mathrm{ml}$ of MYEB for the penicillin biosynthesis assay. Concurrently, a $0.058 \mathrm{ml}$ inoculum of $P$. chrysogenum spore suspension $\left(5.1 \times 10^{7} \mathrm{cfu} / \mathrm{ml}\right)$ in $11.8 \mathrm{ml} \mathrm{PCB}\left(3.0 \times 10^{6} \mathrm{cfu} / \mathrm{PDB}\right)$ was also cultured for three days at $26^{\circ} \mathrm{C}$ in a third PDB (PDB3), before the spent broth was removed with a pipette. The biomass was gathered and compressed using the pipette tip. The approximately cell free supernatant was removed and the process repeated until there was no supernatant. The biomass was retained for the penicillin biosynthesis assay and was submerged in $11.8 \mathrm{ml}$ of MYEB.

The second method was used in the oxytetracycline biosynthesis assay, where two PDB (PDB4 and PDB5) had a $0.5 \mathrm{~mm}$ thick layer $(2.95 \mathrm{ml})$ of silica gel doped with a total of $1.06 \times 10^{9} \mathrm{cfu}$ of $S$. rimosus spores set in the bottom, which was overlaid with $1.0 \mathrm{~mm}$ $(5.9 \mathrm{ml})$ of silica gel. After gelation and aging for three days, $11.8 \mathrm{ml}$ of SRB broth was laid over each of the gels. A third PDB (PDB6) had $11.8 \mathrm{ml}$ of SRB added, which was inoculated with a total of $1.04 \times 10^{9} \mathrm{cfu}$ of $S$. rimosus spore suspension. PDB1-6 were incubated at $26^{\circ} \mathrm{C}\left( \pm 0.1^{\circ} \mathrm{C}\right)$ during the biosynthesis assay without shaking.

\subsection{UV-Irradiation and Sterility Testing}

Gels in PDB1 and PDB4 were UV-irradiated at a distance of $7 \mathrm{~cm}$ from an Oliphant T8/GL30 $30 \mathrm{~W}$ UV-lamp with an emission peak at $254 \mathrm{~nm}$, for various periods. PDB1 was irradiated for $20 \mathrm{~min}$ on the first day and five minutes on each subsequent day. PDB4 was UV-irradiated for 15 min daily. Prior to surface sterilisation, $100 \mu \mathrm{l}$ aliquots of the nutrient broth were removed from each PDB and spread over an MYEA plate. The MYEA plates were incubated at $26^{\circ} \mathrm{C}$ for 3 days and monitored for growth. 


\subsection{Metabolic Analysis}

A $100 \mu \mathrm{l}$ aliquot was removed daily from each PDB for metabolic analysis. The carbohydrate concentration was measured in triplicate against glucose standards using the phenol-sulfuric acid technique [10], modified by the omission of thin-layer chromatographic separation. Penicillin and oxytetracycline concentrations were measured in triplicate by the disc-diffusion assay method [11] using $6 \mathrm{~mm}$ filter disks, each containing $20 \mu \mathrm{l}$ of broth, and B. subtilis as the test organism on PYEA plates incubated at $37^{\circ} \mathrm{C}$ overnight.

\subsection{Use of PDB in Antibiotic Biosynthesis Assays}

The biosynthesis of penicillin was analysed in PDB1-3. Two gels doped with autoclaved $P$. chrysogenum biomass $(2 \% \mathrm{w} / \mathrm{v})$ and two without biomass were used as controls. One of each was UV-irradiated as described above.

The biosynthesis of oxytetracycline was analysed in PDB4-6. All PDB were sampled for sterility and metabolic analysis daily. Samples were taken for 13 days, before the broth was replaced with fresh SRB and testing continued for another 7 days.

\section{Results and Discussion}

The surfaces of UV-irradiated gels containing either $P$. chrysogenum or $S$. rimosus and their overlaying nutrient medium remained sterile for the duration of the experiments (up to 20 days), as demonstrated by the lack of visible surface growth and absence of viable cells in the liquid phase. Consequently, the metabolic activity data from PDB1 and PDB4 were interpreted as being solely due to cells encapsulated within the body of the silica gel matrix. Viable cells were detected in the liquid phases of the PDB that were not UV-irradiated (PDB2 and PDB5) and the majority of the growth appeared to be on the external surface of these gels.

Branyik et al. [3] monitored the outgrowth of cells encapsulated in TEOS-derived gels and polyurethane. Outgrowth from polyurethane and the silica gels was detected within 20 hours of culturing. However, encapsulated cells did not escape from the silica gels over the 35-hour duration of their experiment. Inama et al. [6] reported the successful containment of Saccharomyces cerevisiae in thick films of colloidal silica gel prepared via an aqueous route, by coating the gel with a denser gel layer made via the alkoxide route. How- ever, the denser surface layer inhibited cell function, by inhibiting diffuson of substrate. Campostrini et al. [7] reported the encapsulation of plant cells in a silica matrix composed of a glass mesh and silica gel prepared using an aqueous route. They also used an outer layer formed by the alkoxide route to prevent outgrowth. The colloidal silica used in our work is more porous than silica prepared by acid-catalysed alkoxide routes, and the process of encapsulation is more compatible with the biological functions of cells, as alcohols are not produced during gelation. The UV-sterilization technique reported here prevented the outgrowth of cells for 20 days, making the technique useful for studying metabolic activities of encapsulated cells, without having to encapsulate the cells in silica prepared by the alkoxide route.

The results of the penicillin and oxytetracycline biosynthesis assays are presented in Figs. 1 and 2 and Table 1. The initial concentrations of carbohydrates in the PDB's varied as a result of the volume of the nutrient medium and the volume of water in the gels, which diluted the nutrient medium in PDB1, 2, 4 and 5 by a factor of 0.61 . This gel-water also diluted the resulting products, so yields and yield coefficients were normalised to account for the dilution factor. There was no gel-water in PDB3 and PDB6.

In the penicillin biosynthesis assay, the undoped gels and the gels doped with autoclaved cells (negative

Table 1. Growth parameters of batch cultures of P. chrysogenum and $S$. rimosus growing in and on silica gel and freely suspended in broth (Figs. 1 and 2).

\begin{tabular}{lccc}
\hline PDB & $Y_{P}$ & $Y_{P / S}$ & {$[\mathrm{COH}]_{\mathrm{antib}}$} \\
\hline 1 & 29 & 0.7 & 5.8 \\
2 & 82 & 0.6 & 6.6 \\
3 & 50 & 0.5 & 0.5 \\
$4_{0-13}$ & 62 & $\mathrm{ND}$ & $\mathrm{ND}$ \\
$4_{13-20}$ & 0.0 & 0.0 & $\mathrm{ND}$ \\
$5_{0-13}$ & 726 & 41 & 0.8 \\
$5_{13-20}$ & 102 & 4 & 1.5 \\
$6_{0-13}$ & 779 & 29 & 0.9 \\
\hline
\end{tabular}

PDB: Petri dish bioreactor (PDB1-3 P. chrysogenum in MYEB, PDB4-6 S. rimosus in SRB).

$Y_{P}$ : Total mass of product (penicillin or oxytetracycline) ( $\mu \mathrm{g} /$ bioreactor).

$Y_{P / S}$ : Total mass of product divided by substrate consumed to produce it $(\mu \mathrm{g} / \mathrm{mg})$.

$[\mathrm{COH}]_{\text {antib: }}$ : Carbohydrate concentration where antibiotic biosynthesis began $(\mathrm{mg} / \mathrm{ml})$.

ND: Not determined. 
A)

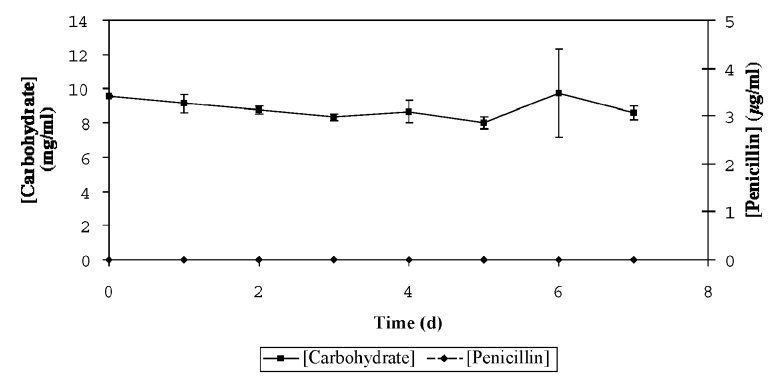

C)

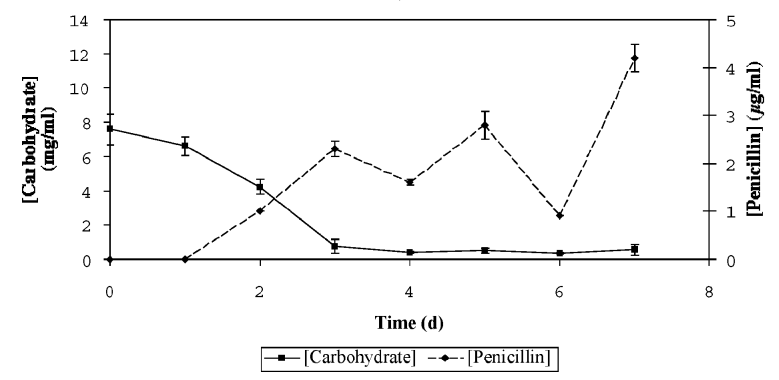

B)

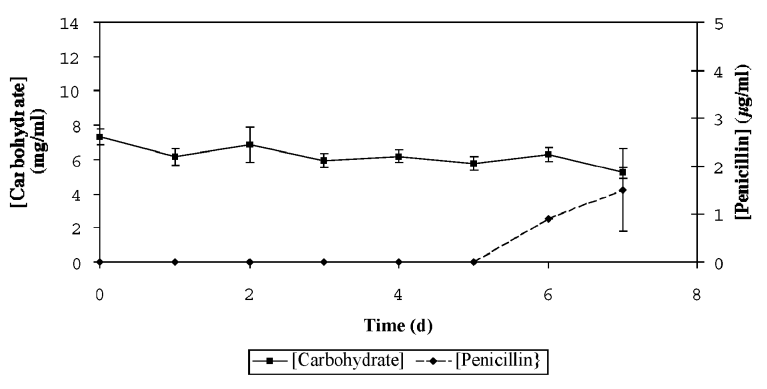

D)

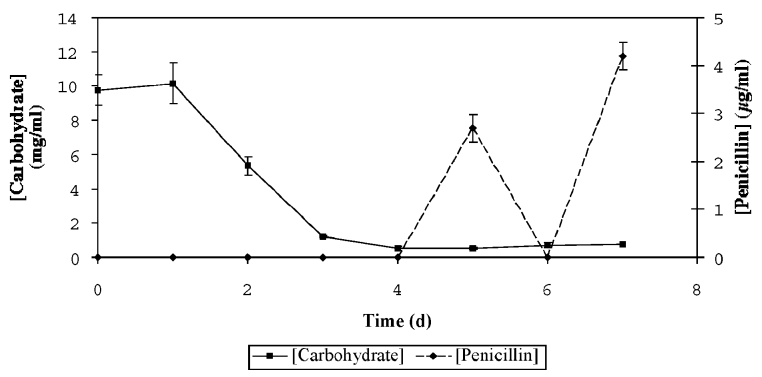

Figure 1. Time course of penicillin production and carbohydrate consumption in various petri dish bioreactors (PDB) by $P$. chrysogenum in batch culture in MYEB medium. (A) Autoclaved P. chrysogenum in silica gel overlaid with MYEB. (B) P. chrysogenum grown in silica gel (UV-irradiated) overlaid with MYEB (PDB1). (C) P. chrysogenum grown on silica gel (not UV-irradiated) overlaid with MYEB (PDB2). (D) $P$. chrysogenum grown freely suspended in MYEB (not UV-irradiated) (PDB3).

controls) appeared to have had little effect on the carbohydrate concentration and produced no penicillin (Fig. 1(A) and data not reported). In contrast, encapsulated and surface-immobilised $P$. chrysogenum produced antibiotic (Fig. 1(B) and (C)). The penicillin yield $\left(Y_{P}\right)$ for cells grown on the gel in PDB2 was significantly greater than that of the encapsulated cells in PDB1 (Table 1), which was most likely due to the slow diffusion of nutrients (particularly dissolved oxygen) through the nanopore network within the latter gel. It should be noted that the carbohydrate had not been completely consumed in PDB1 by day seven and penicillin production had most likely not concluded. The $Y_{P}$ of cells grown freely suspended in liquid medium in PDB3 was slightly less than that for surface-immobilised cells in PDB2 (Table 1).

The substrate-specific antibiotic yield $\left(Y_{P / S}\right)$ in liquid culture (PDB3) was slightly less than that for encapsulated and surface-immobilised cells grown in PDB1 and PDB2, respectively. The lag period before the commencement of penicillin production was longest for encapsulated cells (PDB1), shorter for free cells grown in liquid medium (PDB3) and shortest for surface-immobilised cells (PDB2) (Fig. 1 and Table 1). The concentration of carbohydrate at which penicillin biosynthesis commenced ( $[\mathrm{COH}]_{\text {antib }}$ ) was similar for encapsulated and surface-immobilised cells (PDB1 and PDB2, respectively), and significantly higher than that for free cells grown in liquid medium (PDB3) (Table 1). This was an unexpected result, as the biofilm that grew on the surface of the gel was bathed in liquid medium (hence the gel could not have limited the availability of nutrients as would be the case for encapsulated cells), and thus was expected to exhibit similar bioactivity to cells grown freely suspended in liquid medium.

Relatively little oxytetracycline was produced by encapsulated S. rimosus in PDB4 during the first batch and none was produced during the second batch with reused biomass (Fig. 2(A) and (B) and Table 1). When $S$. rimosus was grown on the gel (PDB5), the observed value of $Y_{P}$ (Fig. 2(C)) was only slightly less than that for cells grown freely suspended in liquid medium (Fig. 2(E) and Table 1). Very little oxytetracycline was produced by $S$. rimosus grown on the gel in the second batch (Fig. 2(D)), which may have been due to metabolic "memory", which prevents 


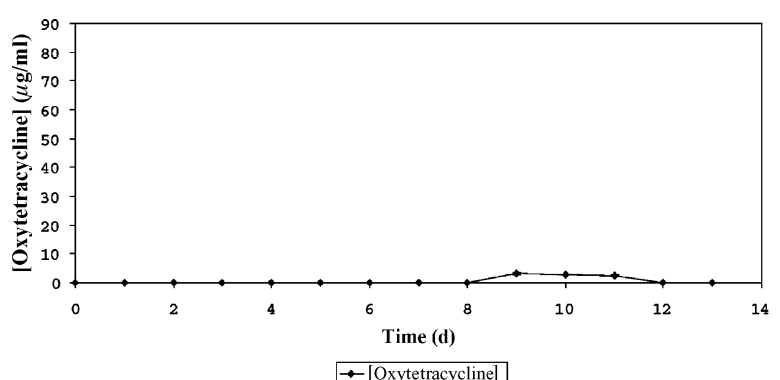

C

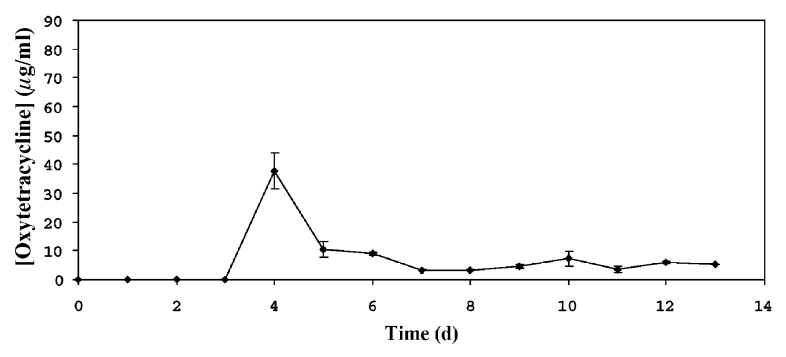

$\rightarrow-[$ Oxytetracycline]

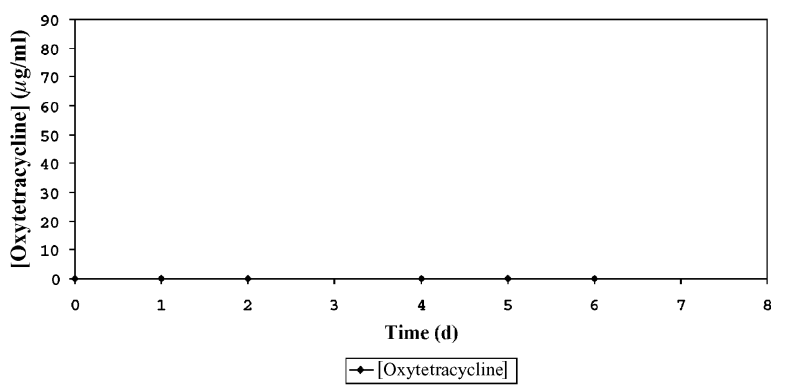

D

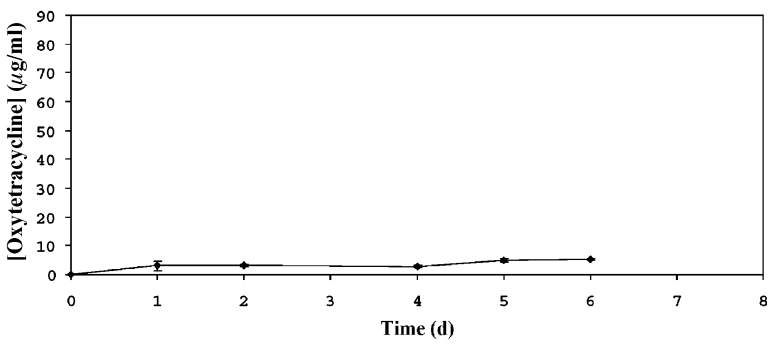

$\rightarrow[$ Oxytetracycline]

$\mathbf{E}$

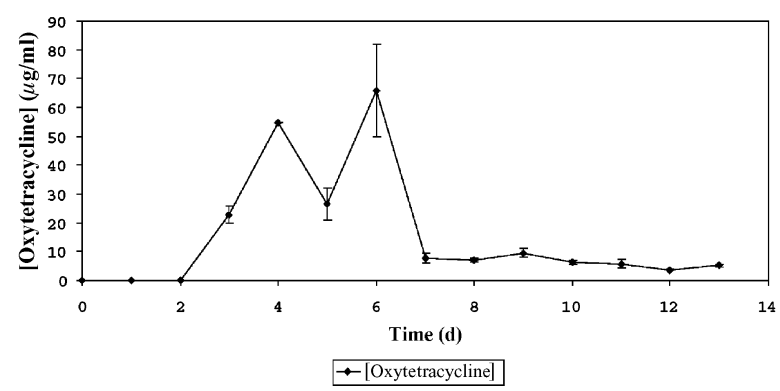

Figure 2. Time course of oxytetracycline production and carbohydrate consumption in various petri dish bioreactors (PDB) by S. rimosus in batch culture in SRB medium. (A) S. rimosus grown in silica gel (UV-irradiated) overlaid with SRB (PDB4 $0-13$ ). (B) The same gel as in A) overlaid with fresh SRB on day 13 (PDB4 ${ }_{13-20}$ ). (C) S. rimosus grown on silica gel (not UV-irradiated) overlaid with SRB (PDB5 $0-13$ ). (D) The same gel as in C) overlaid with fresh SRB on day 13 (PDB5 $13-20$ ). (E) S. rimosus grown freely suspended in SRB (not UV-irradiated) (PDB6 $0-13$ ).

S. rimosus cells from resuming antibiotic biosynthesis after they have initially ceased production. The pattern of antibiotic biosynthesis kinetics of encapsulated and surface-immobilised $S$. rimosus was similar to that of the corresponding $P$. chrysogenum samples. The lag for oxytetracycline production for encapsulated $S$. rimosus (PDB4) was longer than for surface-immobilised cells (PDB5), although the lag for the onset of oxytetracycline production was shortest for cells grown in liquid medium. The carbohydrate concentration where oxytetracycline production began $\left([\mathrm{COH}]_{\mathrm{antib}}\right)$ for encapsu- lated and surface-immobilised S. rimosus was similar to that for cells grown freely suspended in liquid medium (Table 1).

\section{Conclusions}

The UV-sterilisation technique was an effective technique for preventing the outgrowth of cells from inoculated gels, which made it possible to differentiate the activities of encapsulated and surface-immobilised cells. 
Carbohydrate consumption and antibiotic biosynthesis were observed for both encapsulated P. chrysogenum and $S$. rimosus, demonstrating that the gels were sufficiently porous to permit the required diffusion of oxygen and substrates. However, the yields and kinetics were relatively poor compared with surfaceimmobilised cells or cells freely suspended in broth. Efforts to tailor the sol gel chemistry to maximise the porosity of the silica gel could also enhance the productivity of encapsulated cells.

\section{Acknowledgments}

We thank E. Drabarak, Dr K. Short and D. Cassidy for their assistance.

\section{References}

1. Karube, S. Suzuki, and E.J. Vandamme, in Biotechnology of
Industrial Antibiotics, edited by E.J. Vandamme (Marcel Dekker, New York, 1984), Vol. 22 p. 761.

2. S. Diré, A. Cavazza, R. Campostrini, and G. Carturan, Europ. Mater. Res. Sci. Monog. 5, 151 (1992).

3. T. Branyik, G. Kuncova, J. Paca, and K. Demnerova, J. Sol-Gel Sci. Technol. 13, 283 (1998).

4. K.S. Finnie, J.R. Bartlett, and J.L. Woolfrey, J. Mater. Chem. 10, 1099 (2000).

5. N. Nassif, O. Bouvet, M.N. Rager, C. Roux, T. Coradin, and J. Livage, Nature Materials 1, 42 (2002).

6. L. Inama, S. Dire, G. Carturan, and A. Cavazza, J. Biotechnol. 30, 197 (1993).

7. R. Campostrini, G. Carturan, R. Caniato, A. Piovan, R. Filippini, G. Innocenti, and E.M. Cappelletti, J. Sol-Gel Sci. Technol. 7(1/2), 87 (1996).

8. S.S. Yang and S.S. Yaun, World J. Microbiol. Biotechnol. 6, 236 (1990).

9. S.S. Yang, L. Chiu, and S.S. Yaun, World J. Microbiol. Biotechnol. 10, 215 (1990).

10. M. Dubois, K.A. Gilles, J.K. Hamilton, P.A. Rebers, and F. Smith, Analy. Chem. 28, 350 (1956).

11. A. Bauer, W.M.M. Kirby, J.C. Sherris, and M. Turck, Am. J. Clin. Path. 45(4), 493 (1966). 